\title{
Data-driven parameterized modeling of LTI systems with guaranteed stability
}

\author{
S. Grivet-Talocia, T. Bradde, M. De Stefano, and A. Zanco \\ Dept. of Electronics and Telecommunications, Politecnico di Torino, Italy
}

\section{Outline}

- Main objective: automated extraction of parameterized ROMs from sampled frequency responses, for Electronic Design Automation

- Requirements: models must be stable in the parameter space, enabling transient numerical simulations during design flow

- Problem: state-of-the-art techniques may not guarantee model stability

- This work: we present a new framework based on a parameterized Sanathanan-Koerner iteration with stability constraints

- Main result: a sufficient condition and an algorithm for extracting guaranteed stable ROMs from sampled responses

- Enabling technologies: Hamiltonian pencil eigenvalue perturbation for adaptive construction of stability constraints, enforcing positive realness of denominator submodel

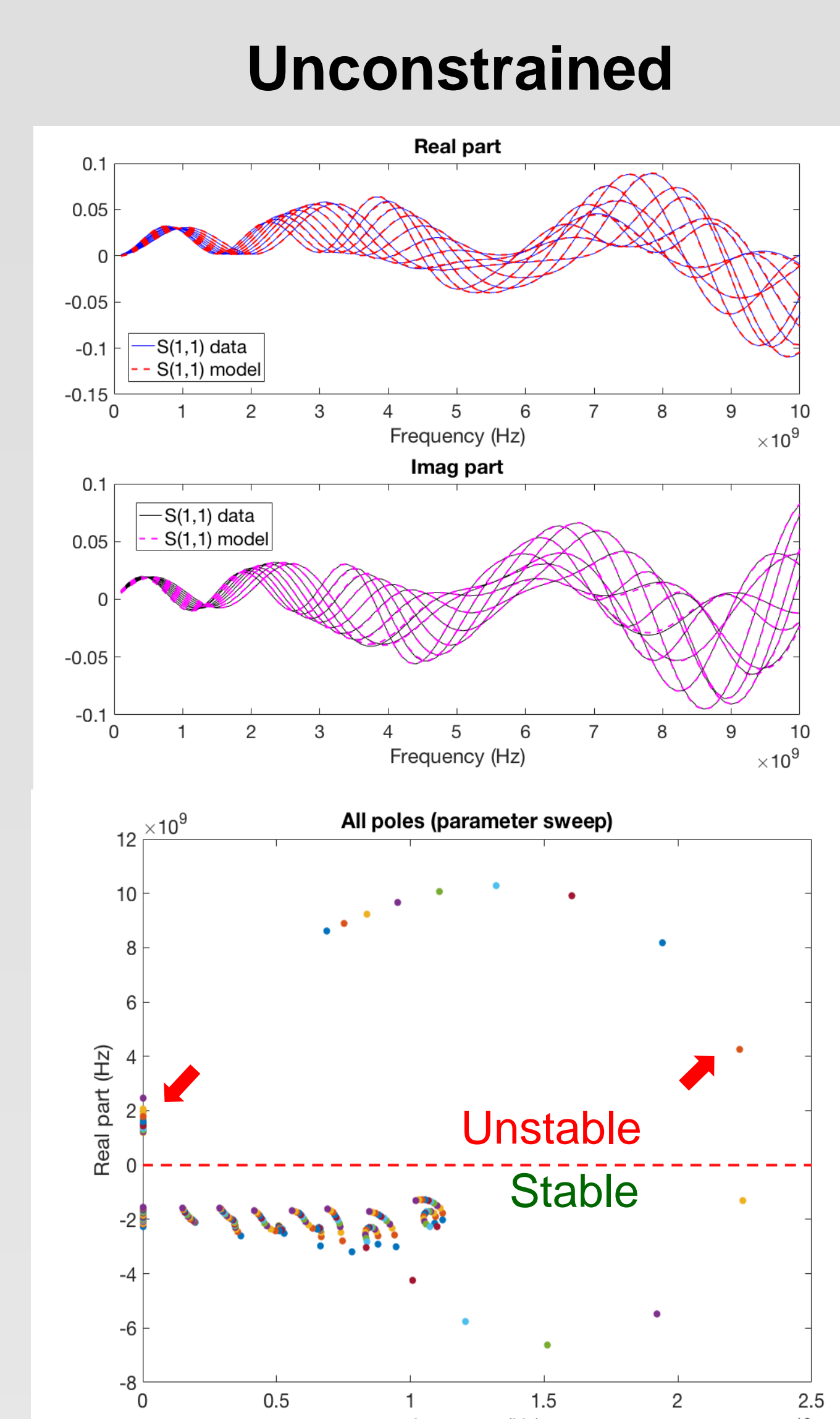

Proposed approach

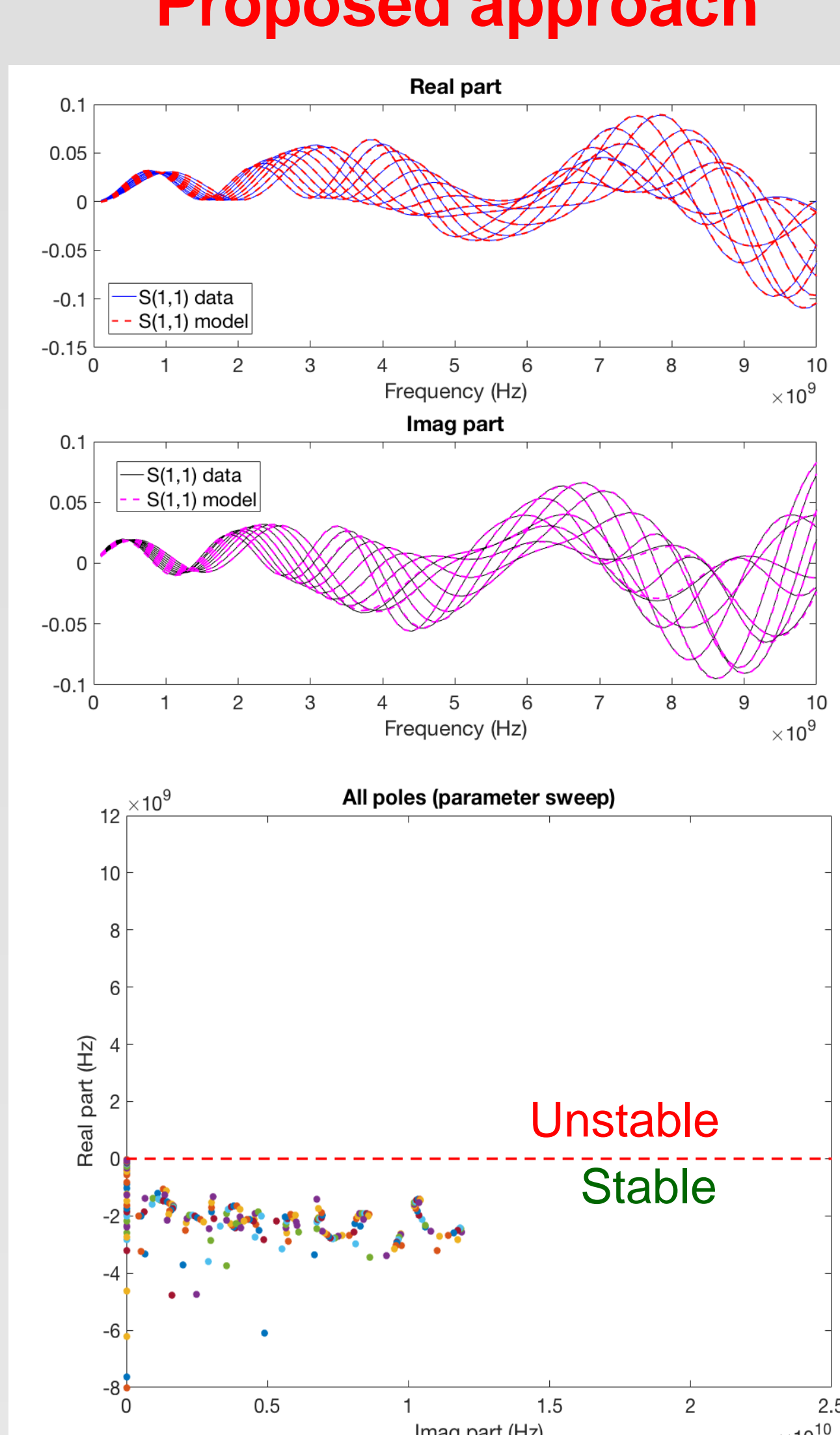

\section{Model structure and identification}

Input data samples: $\breve{\mathbf{H}}_{k, m}=\breve{\mathbf{H}}\left(s_{k} ; \boldsymbol{\vartheta}_{m}\right)$

Model: parameterized Sanathanan-Koerner form

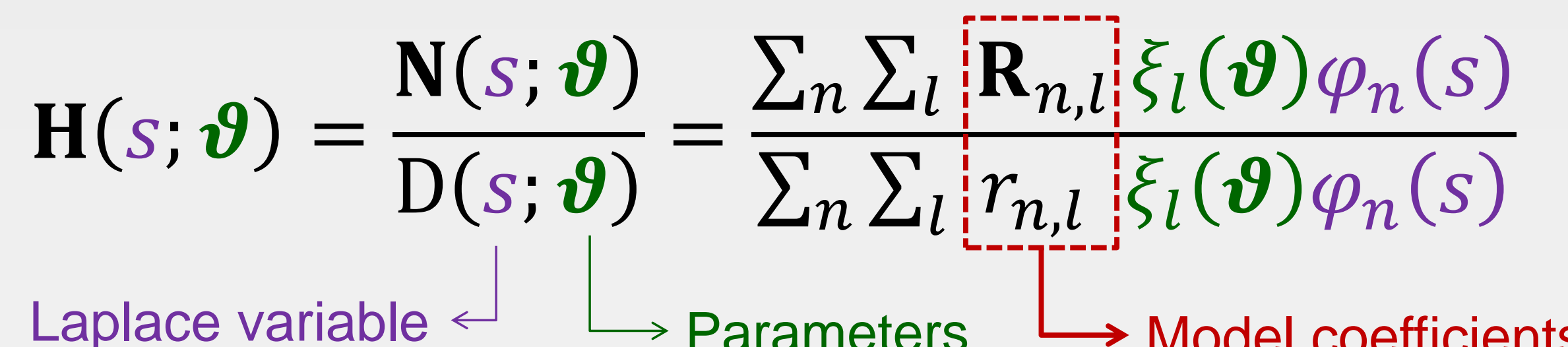

$\xi_{l}(\boldsymbol{\theta}) \quad$ Parameter-space basis functions multivariate orthogonal polynomials mixed Fourier + polynomials; any other choice...

Frequency-domain basis functions: partial fractions

$$
\varphi_{0}(s)=1, \quad \varphi_{n>0}(s)=\left(s-q_{n}\right)^{-1} \quad \text { "basis poles", fixed }
$$

Fit model to data: $\quad \mathbf{H}\left(s_{k} ; \boldsymbol{\vartheta}_{m}\right)=\frac{\mathbf{N}\left(s_{k} ; \boldsymbol{\vartheta}_{m}\right)}{\mathrm{D}\left(s_{k} ; \boldsymbol{\vartheta}_{m}\right)} \approx \breve{\mathbf{H}}_{k, m}$ Parameterized GSK iteration

$D_{0}=1$

for $\mu=1,2, \ldots$

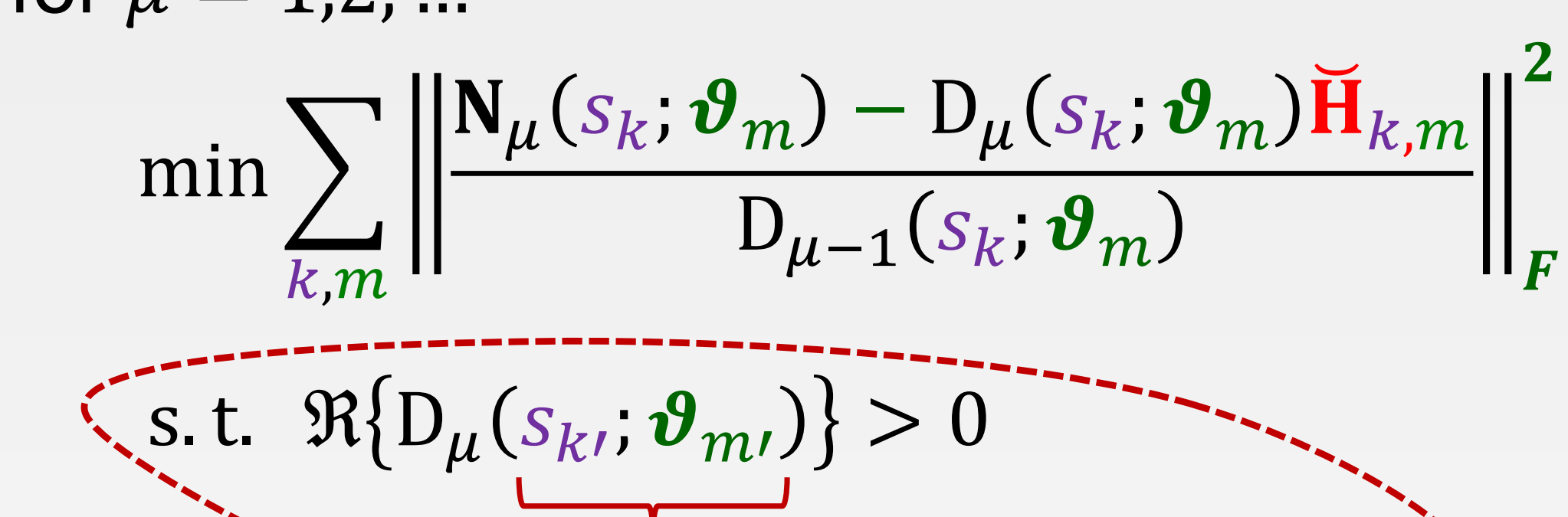

$$
\begin{array}{ll}
\mathrm{D}(s ; \boldsymbol{\vartheta}): & \dot{\boldsymbol{w}}=\boldsymbol{A} \boldsymbol{w}+\boldsymbol{B} \boldsymbol{u} \\
& \boldsymbol{y}=\boldsymbol{C}(\boldsymbol{\vartheta}) \boldsymbol{w}+\boldsymbol{D}(\boldsymbol{\vartheta}) \boldsymbol{u}
\end{array}
$$

end

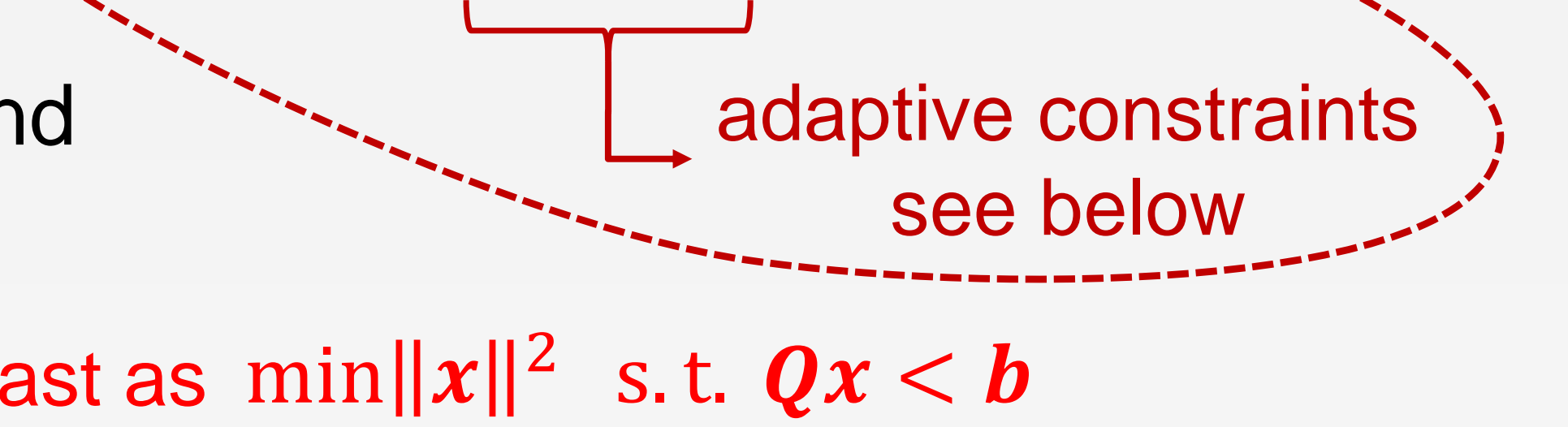

Cast as $\min \|x\|^{2}$ s.t. $\boldsymbol{Q} x<\boldsymbol{b}$

\section{Key result: building constraints for uniform stability}

Theorem: if $\mathrm{D}(s ; \boldsymbol{\vartheta})$ is a (strictly) Positive-Real function, then $\mathfrak{R}\left\{p_{n}(\boldsymbol{\vartheta})\right\}<0, \forall \boldsymbol{\vartheta}$

Building adaptive constraints

1. $\mathrm{D}(s ; \vartheta)$ regular for $\mathfrak{R}\{s\}>0$

$\longrightarrow$ guaranteed by

3. $\mathfrak{R}\{\mathrm{D}(s ; \vartheta)\} \geq \varepsilon>0$ for $\mathfrak{R}\{s\}>0 \longrightarrow$ check for $s=j \omega$

Hamiltonian pencil $(\boldsymbol{M}(\boldsymbol{\vartheta})-\lambda \boldsymbol{K})$ associated to denominator submodel

$$
\boldsymbol{M}(\boldsymbol{\vartheta})=\left[\begin{array}{ccc}
\boldsymbol{A} & \mathbf{0} & \boldsymbol{B} \\
\mathbf{0} & -\boldsymbol{A}^{T} & -\boldsymbol{C}^{T}(\boldsymbol{\vartheta}) \\
\boldsymbol{C}(\boldsymbol{\vartheta}) & -\boldsymbol{B}^{T} & \boldsymbol{D}(\boldsymbol{\vartheta})+\boldsymbol{D}^{T}(\boldsymbol{\vartheta})
\end{array}\right] \quad \boldsymbol{K}=\left[\begin{array}{lll}
\boldsymbol{I} & \mathbf{0} & \mathbf{0} \\
\mathbf{0} & \boldsymbol{I} & \mathbf{0} \\
\mathbf{0} & \mathbf{0} & \mathbf{0}
\end{array}\right]
$$

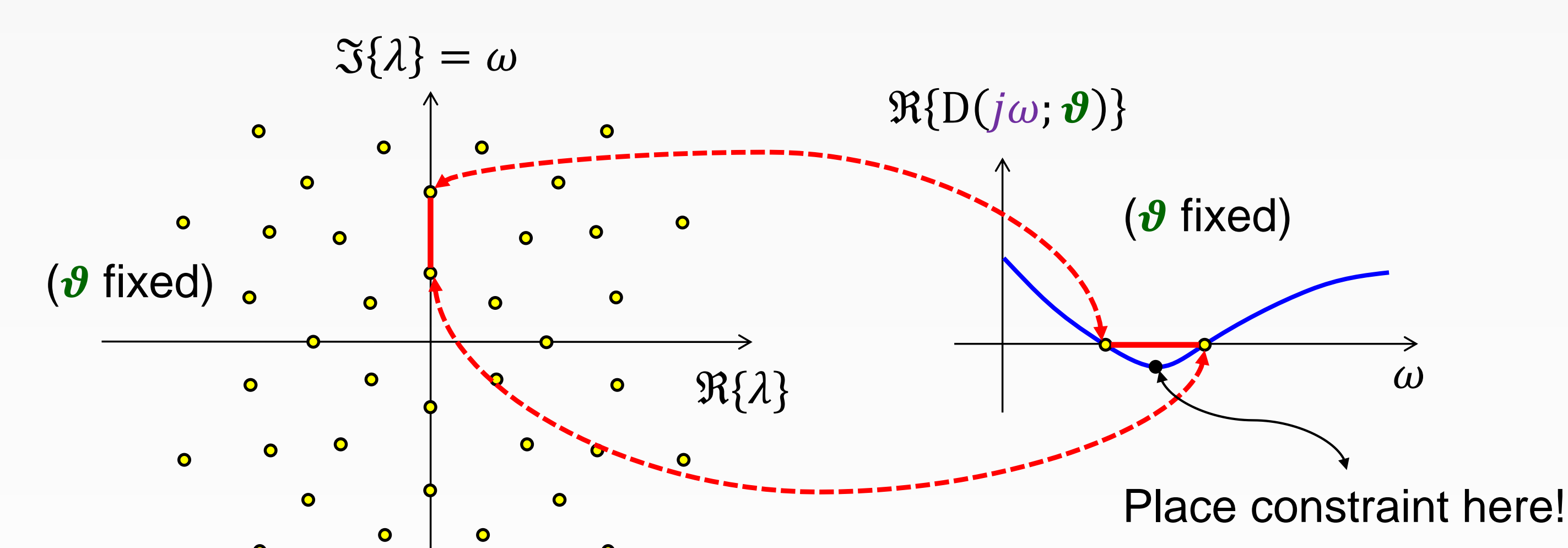

Hamiltonian pencil $(\boldsymbol{M}(\boldsymbol{\vartheta})-\lambda \boldsymbol{K})$ eigs $\quad$ Real part of $\mathrm{D}(j \omega ; \boldsymbol{\vartheta})$

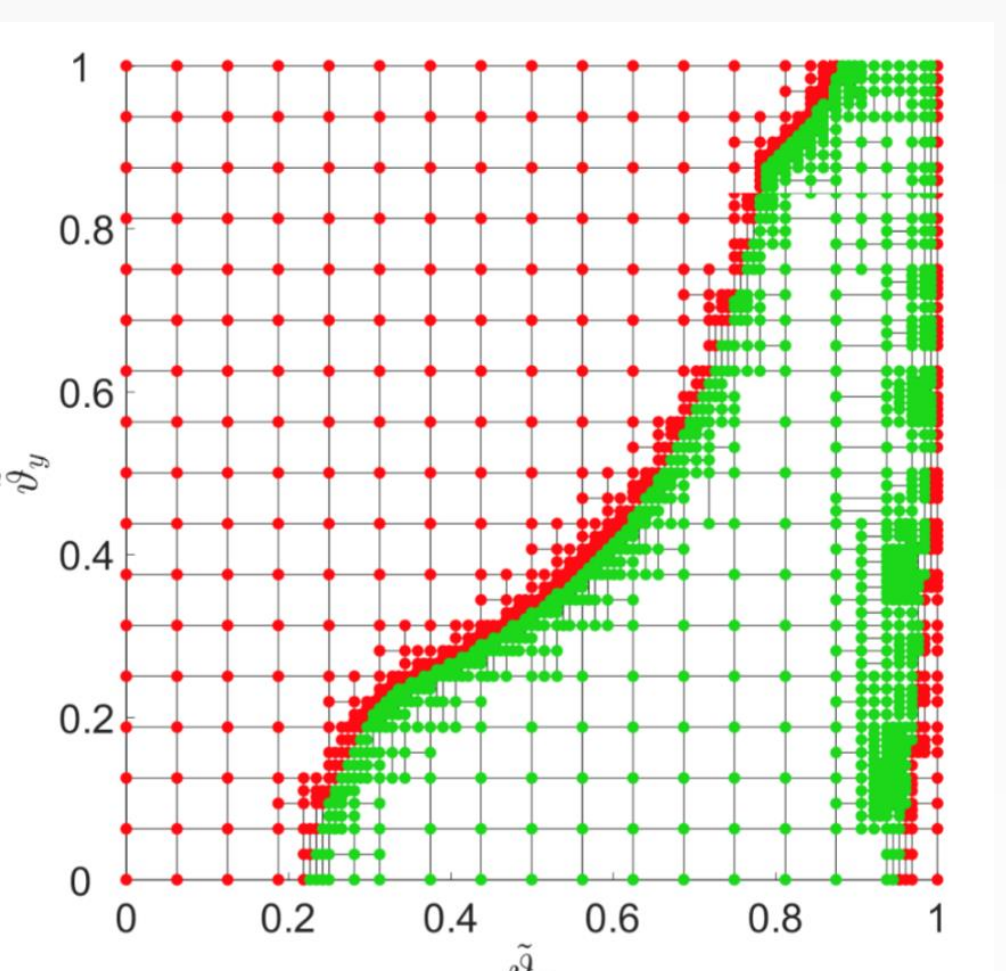

Adaptive sweep on $\vartheta$

Bottom line: passivity of denominator submodel implies uniform model stability $\rightarrow$ Let us enforce denominator passivity!

S. Grivet-Talocia, "A Perturbation Scheme for Passivity Verification and Enforcement of Parameterized Macromodels," IEEE Trans. on CPMT, Vol. 7, No. 11, Nov. 2017, pp.1869-1881

\section{Examples}

A reverberation chamber

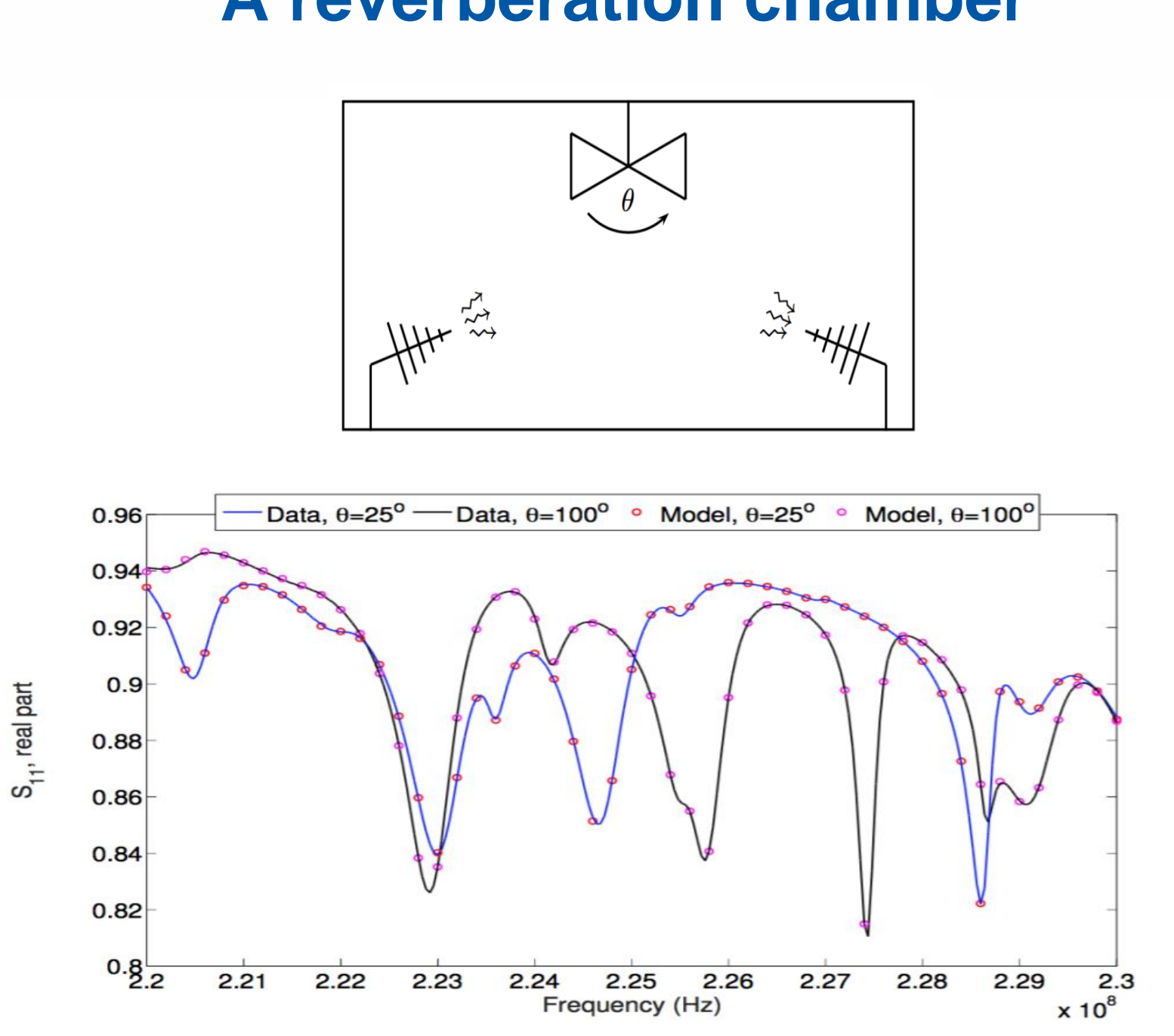

An integrated inductor

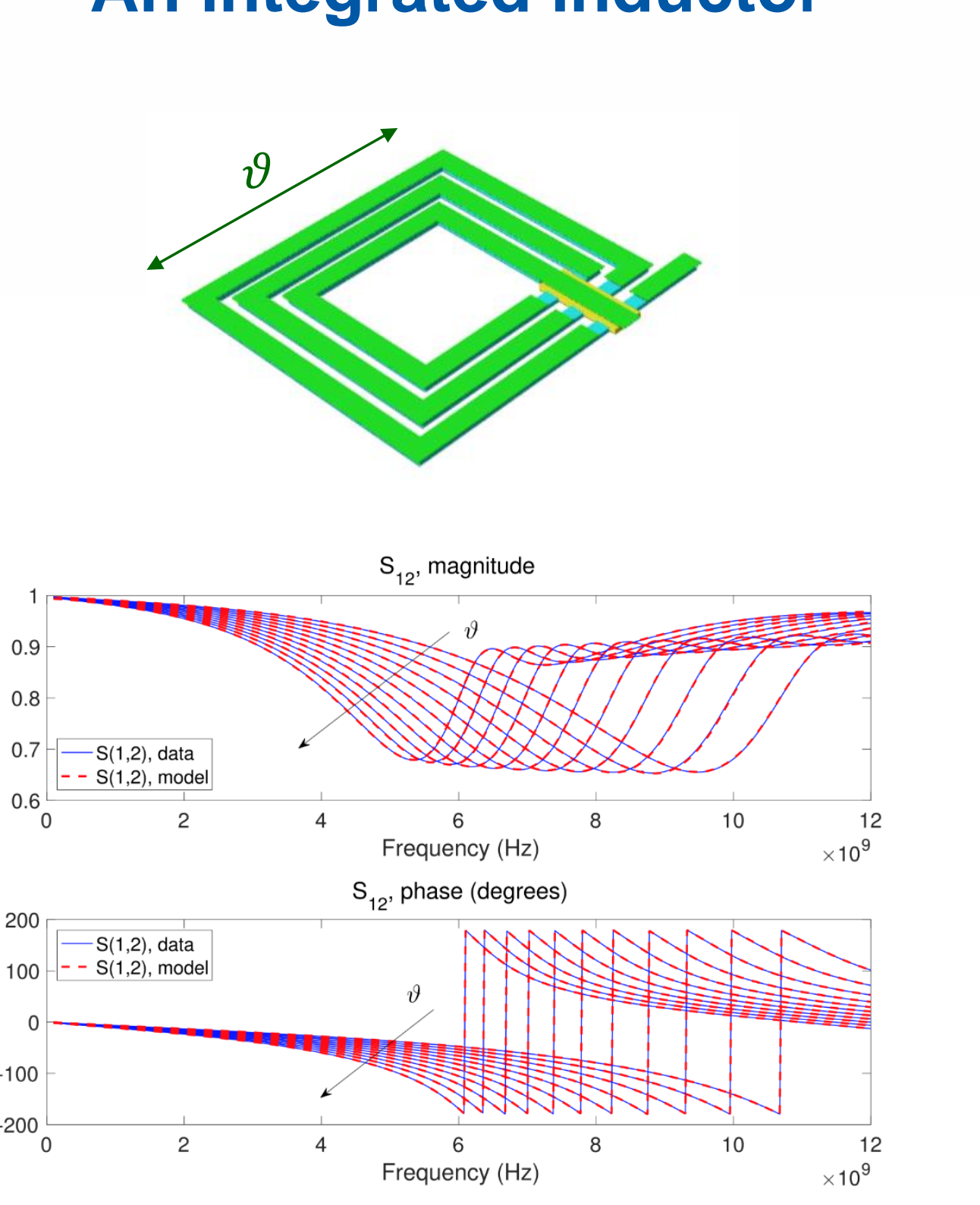

A complex Printed Circuit Board interconnect
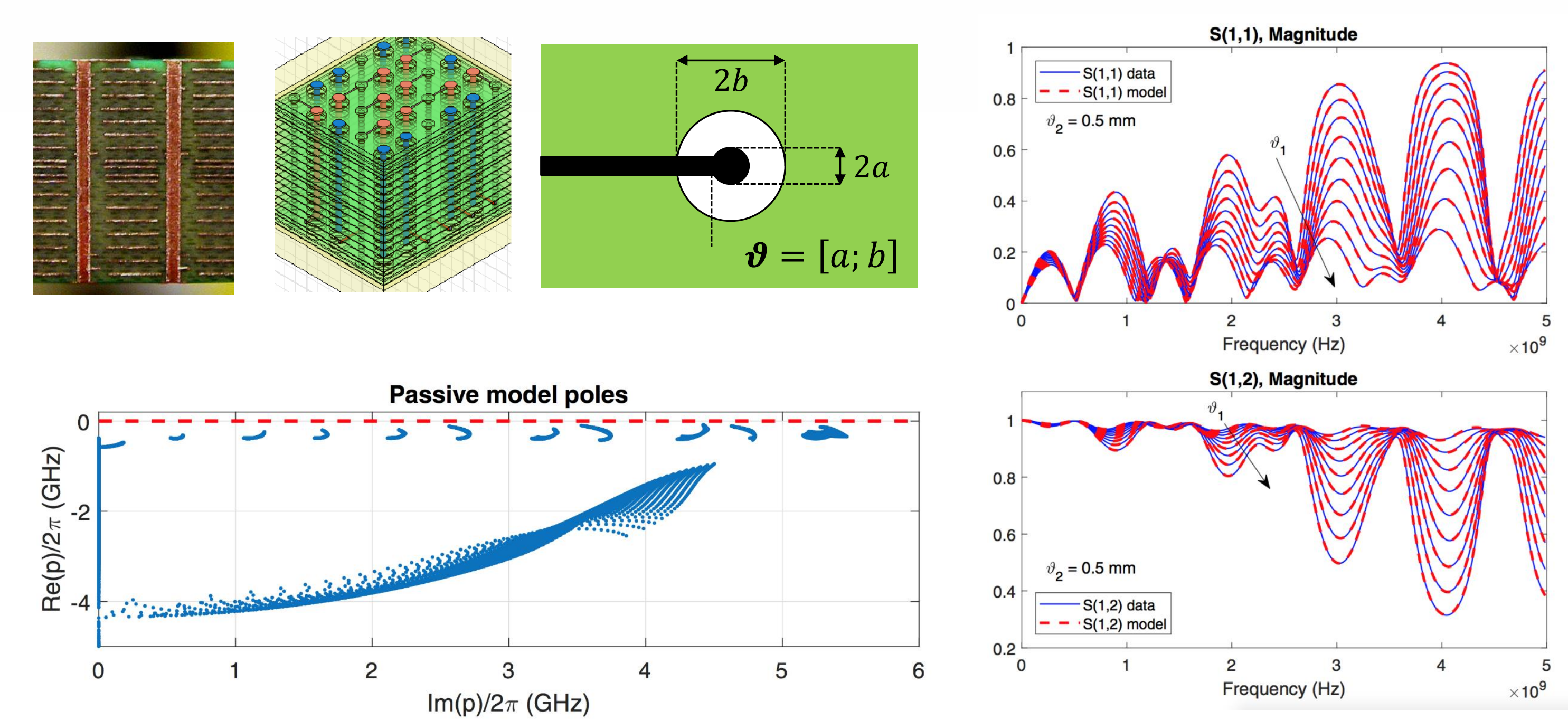